\title{
Betriebsnachfolge achtsam gestalten - die Rolle des systemischen Nachfolge-Coachings in Unternehmerfamilien am Beispiel des ****Hotel Alpenglühen
}

\author{
Anita Zehrer ${ }^{1} \cdot$ Gabriela Leiß $^{1}$ \\ Online publiziert: 30. Juli 2018 \\ (c) Der/die Autor(en) 2018
}

\section{Zusammenfassung}

Unternehmerfamilien sind komplexe Systeme, geprägt durch wechselseitige Einflüsse und Rückkoppelungen in den beiden Subsystemen Familie und Unternehmen. Eine wesentliche Herausforderung stellt die Betriebsnachfolge dar, der in vielen Unternehmen zu wenig Beachtung geschenkt wird und die durch systemisches Nachfolge-Coaching begleitet werden sollte. Der folgende Beitrag beschreibt daher die Besonderheiten von Unternehmerfamilien sowie die Betriebsnachfolge als zentrale Herausforderung und präsentiert im Anschluss ein Fallbeispiel. In gegenständlichem Fall werden die dort eingesetzten Gesprächsformate - Einzelgespräche, Paargespräche und Familienworkshops - sowie angewandten Coaching-Methoden Zielarbeit und Ökologie-Check, Visionsarbeit, Nachfolge-Landkarte - beschrieben, zu Arbeitshypothesen verdichtet und in ein daraus abgeleitetes Vorgehensmodell für ein systemisches Nachfolge-Coaching überführt.

Schlüsselwörter Familienunternehmen · Systemisches Coaching $\cdot$ Betriebsnachfolge $\cdot$ Nachfolge-Coaching · Gesprächsformate $\cdot$ Fallbeispiel

\section{Mindful Family Firm Succession-the Role of Systemic Succession Coaching in Entrepreneurial Families on the Example of the ****Hotel Alpenglühen}

\begin{abstract}
Entrepreneurial families are complex systems, characterized by mutual influences and reactions of both systems-family and business. One essential challenge is business succession, which receives little attention in many family firms and would need systemic succession coaching along the process. This paper thus describes peculiarities of entrepreneurial families and the challenge of business succession. By means of a case study and herein applied conversation formats-single coaching, pair coaching and family workshops—as well as coaching methods—goal setting and ecology check, visionalizing, succession map-working hypotheses and a process model for systemic succession coaching are introduced.
\end{abstract}

Keywords Family business $\cdot$ Systemic coaching $\cdot$ Business succession $\cdot$ Succession coaching $\cdot$ Coaching formats $\cdot$ Case study

Publisher's Note Springer Nature remains neutral with regard to jurisdictional claims in published maps and institutional affiliations.

Anita Zehrer

anita.zehrer@mci.edu

Gabriela Leiß

gabriela.leiss@mci.edu

1 Zentrum Familienunternehmen,

Universitätsstraße 15, 6020 Innsbruck,

Österreich 


\section{Einleitung}

Familienunternehmen gibt es in unterschiedlichen Größen und Organisationsformen, vom kleinen mittelständischen Betrieb bis hin zum multinationalen Konzern (Klein 2000, 2004). „Familienunternehmen sind zunächst einmal Unternehmen. Das bedeutet, dass sie sich den Regeln und der Logik der Wirtschaft zu unterwerfen haben" (Lippmann 2013, S. 5). Familien funktionieren allerdings nach anderen Prinzipien als Unternehmen. Unternehmerfamilien sind „keine Unternehmen, aber sie sind auch nicht nur und ausschließlich Familie. Sie sind Familien, in deren Umgebung sich dauerhaft ein Unternehmen befindet" (von Schlippe et al. 2017, S. 99). Die Systemtheorie dient als analytischer Bezugsrahmen für das bessere Verständnis der Komplexität einer Unternehmerfamilie und der Auswirkungen auf die beiden Subsysteme Familie sowie Unternehmen (Luhmann 1995; Tagiuri \& Davis 1996; Simon 2009). Dabei gilt es, Systemzusammenhänge, wechselseitige Einflüsse und Rückkoppelungen innerhalb einer Unternehmerfamilie zu betrachten.

Die Unternehmensübergabe bzw. -nachfolge stellt für Familienunternehmen eine kritische Phase dar (CabreraSuárez et al. 2001; Lee et al. 2003; Spelsberg und Weber 2012). Nur ein kleiner Teil der Unternehmen bewältigt die familieninterne Übergabe über mehrere Generationen (Kets de Vries 1993; Davis und Harveston 1999; Scholes et al. 2008; Klein et al. 2010). Laut Untersuchungen schaffen nur bis zu $66 \%$ der Unternehmen den Sprung in die zweite Generation. Bei der Weitergabe in die dritte Generation sinkt der Prozentsatz sogar auf 10-37\% (Hennerkes et al. 2007). Studien zufolge gibt es europaweit etwa 610.000 Unternehmen, welche vor den Herausforderungen der Nachfolge stehen (Halter 2009; Klein et al. 2010; Halter und Schröder 2011;); allerdings wird der Unternehmensübergabe in vielen Unternehmen zu wenig Beachtung geschenkt (Zellweger et al. 2012).

Im Hinblick auf das Coaching von Familienunternehmen stellt sich die Frage, ob deren Besonderheiten sich auch im Nachfolge-Coaching niederschlagen, was laut Shams (2011) nach wie vor eine Forschungslücke darstellt. „Despite the high percentage of family businesses among all businesses in the world, there is still a lack of consolidated literature on family business coaching practices, which may well be because family business coaching has been taken for granted and the need to develop a solid theoretical base with practical applications, on which family business coaching can be grounded, has not been sufficiently appreciated" (Shams 2011, S. 6). Da derzeit kein Coaching-Modell für Unternehmerfamilien besteht, unternimmt dieser Beitrag den Versuch, ein Vorgehensmodell zum systemischen Nachfolge-Coaching von Unternehmerfamilien samt Coaching-Methoden und Instrumenten aufzustellen und damit folgende Forschungslücken zu schließen: „Therefore, increasing attention should be focused on the development of generic and specific family business coaching skills ... A collective effort from academics, practitioners, coaches, and businesses can ensure continuity in delivering good coaching practices for family businesses ..." (Shams 2011, S. 10). Da in Familienunternehmen die Systeme Familie und Unternehmen derart stark verschmelzen, stellt sich gerade bei der Nachfolge die Frage, wie systemisches Business-Coaching im Prozess der Betriebsübergabe sinnvoll eingesetzt werden kann.

Der Beitrag beschreibt daher zunächst die Besonderheiten von Unternehmerfamilien sowie die Betriebsnachfolge als eigene Herausforderung und präsentiert dann ein Fallbeispiel. Fallstudien sind vor allem bei Forschungen im Bereich der Familienunternehmen aufgrund der Heterogenität der einzelnen Unternehmen sehr beliebt und auch notwendig (Pounder 2015; Goel und Jones 2016; Leppa et al. 2016). Der dokumentierte Fall ist ein traditionelles touristisches Familienunternehmen. In gegenständlichem Fall werden die dort eingesetzten Gesprächsformate - Einzelgespräche mit den sechs Familienmitgliedern, Paargespräche mit den drei Paaren davon ein Übergeberpaar und zwei Übernehmerpaare sowie ein Familienworkshop mit allen Familienmitgliedern - beschrieben und zu Arbeitshypothesen verdichtet. Nach einer Einführung zum Fallbeispiel werden die Erkenntnisse und Schlüsselerlebnisse in den einzelnen Gesprächsformaten beschrieben sowie am Ende das daraus abgeleitete Vorgehensmodell für ein systemisches Nachfolge-Coaching vorgestellt. Zusammenfassend wird folgenden Forschungsfragen nachgegangen:

1. Welche Besonderheiten sind beim intergenerationalen Wechsel in Familienunternehmen zu berücksichtigen?

2. Wie lässt sich ein systemischer Nachfolge-CoachingProzess bei Unternehmerfamilien strukturieren?

3. Welche funktionalen Gesprächsformate lassen sich im Nachfolge-Coaching von Unternehmerfamilien anwenden?

\section{Die Unternehmerfamilie als System}

Ein wesentliches Merkmal von Unternehmerfamilien liegt in der engen Verzahnung von Familie, Betrieb und Eigentum, wobei ,jedes dieser sozialen Gebilde [...] seine charakteristische Eigendynamik [besitzt], die jedoch von den anderen mitgeprägt wird, ohne dass diese ihre Eigenlogik damit einbüßen würden“"(Wimmer et al. 2009, S. 102). Systemtheoretisch lassen sich Unternehmerfamilien als ,soziale Systeme unterschiedlichen Typs" (Simon 2009, S. 19) beschreiben, die sich nur durch Kommunikation aufrechterhalten und voneinander abgrenzen (Simon 2007, S. 88), 
wobei die Differenzierung über jeweils eigene und teilweise widersprüchliche Kommunikationsmuster und -regeln erfolgt. So ist familiäre Kommunikation in der Regel stark personenorientiert, direkt und spontan, wenig formalisiert und flexibel. Im Mittelpunkt stehen Beziehung, Loyalität und Wertschätzung, Sinnstiftung, Sicherheit und Vertrauen. Die Zugehörigkeit zur Familie ist dem Grunde nach unkündbar, Rollen können sich im Laufe des Lebens ändern. Im Unternehmen stehen dagegen Leistung und Output im Vordergrund mit dem Ziel, reibungslose und personenunabhängige Abläufe und Prozesse und damit das Überleben des Betriebs sicherzustellen. Die zumeist schriftliche Kommunikation ist deshalb in hohem Maße sachbezogen und formalisiert. Geld dient als zentrale Währung. Die Zugehörigkeit zum Unternehmen ist vertraglich begründet und kann bei Bedarf jederzeit beendet werden (Simon et al. 2005; Simon 2005, 2009; Leiß und Zehrer 2018b).

Die klassische Rollenanalyse und in weiterer Folge die Entwicklung der Rollentheorie schaffen die Möglichkeit, soziale Sachverhalte zu erklären und zu beschreiben. Basierend auf der systempsychologischen Rollentheorie nach Mead und Linton (Stadler und Kern 2010) übernimmt in Unternehmerfamilien jedes Mitglied unterschiedliche Rollen in diesen drei Subsystemen mit schwer zu vereinbarenden Zuschreibungen und Erwartungen, sodass Entscheidungen gleichermaßen von sachlichen und emotionalen Motiven geleitet werden (Rüsen 2009). Das Rollenverhalten umfasst Entscheidungen und Handlungen einzelner Rollenträger (Jackson 1972). Die Forschung in diesem Bereich schafft die Voraussetzung, diese Sachverhalte deutlicher, wirklichkeitsnäher und/oder angemessener deskriptiv zu erfassen (Wiswede 1977). Es wird von jedem Individuum ein bestimmtes Rollenverhalten erwartet. Wenn der Rollenträger diese Erwartungen nicht erfüllt und sich nicht regelkonform verhält, können Ablehnung und Zurückweisung die Folge sein (Sipos 2012). Die Analyse von Rollenkonflikten ist eines der zentralen Themen der Rollentheorie (Wiswede 1977). Da die Grenze zwischen Familie und Betrieb in den meisten Fällen unscharf ist, kommt es zu einer ständigen Rollenvermischung mit entsprechenden Kommunikationsparadoxien wie zum Beispiel „Sei gleichzeitig ein fürsorglicher Vater und ein verantwortungsvoller Unternehmer!“ oder „Sei gleichzeitig gerecht in beiden Systemen!“ (von Schlippe et al. 2008, S. $24 \mathrm{ff}$.). Die nicht auflösbare Unvereinbarkeit von sich ausschließenden Werten und Zielen und daraus resultierende Konflikte gehören zu den zentralen Herausforderungen für alle Beteiligten. In der Praxis finden sich verschiedene Bewältigungsstrategien im Umgang mit diesen hochexplosiven, sich langsam füllenden „Nitroglyzerin-Fläschchen“ (von Schlippe 2009, S. 43), die von Tabuisierung bis zum reflektierten Ausbalancieren reichen.

\section{Die Nachfolge als besondere Herausforderung für Unternehmerfamilien}

Der Anteil an Familienunternehmen und damit deren ökonomischer Beitrag ist enorm (Harvey und Evans 1995, 1994): Geschätzte $80 \%$ aller Unternehmen in der EU sind in Familienhand und stellen zusammen rund $60 \%$ der Arbeitsplätze im Privatsektor (EU 2015). Der Wechsel von einer Generation zur nächsten gilt als besonders kritische Phase (Cater \& Justis 2009; Nordqvist et al. 2013), die für die Überlebensfähigkeit von Familienunternehmen von zentraler Bedeutung ist und auch gesamtwirtschaftlich eine große Bedeutung hat.

Bereits in den 1980/90er-Jahren beschäftigen sich Lansberg (1988) und Harveston et al. (1997) mit der Problematik der Unternehmensweitergabe - im Fokus der Ausarbeitungen steht allerdings stets der Übergebende (Handler 1994), wohingegen sich gegenwärtige Forschung vermehrt mit dem Nachfolger beschäftigt (De Massis et al. 2008; Brückner 2011; Collins 2011; Prügl und Hauck 2012). Auf Nachfolgerseite geht es hierbei unter anderen Fähigkeiten um das Wissen (De Massis et al. 2008; Humphreys 2013), aber auch Werte, Persönlichkeitszüge und Führungsstile (De Massis et al. 2008; Ghee et al. 2015; Miller et al. 2003; Mussolino und Calabro 2014). Eine besondere Rolle nehmen auch Faktoren wie Bereitschaft, Bewusstsein, Macht und Rollenverhältnisse ein (De Massis et al. 2008; Humphreys 2013; Sund et al. 2015). Diese münden unter Umständen in intergenerative Differenzen zwischen Übergeber und Übernehmer (Burböck und Krenn 2014; Davis und Harveston 1998; Schlepphorst und Moog 2014). Positive oder negative Auswirkungen können auch durch das Kommunikationsverhalten, die Rollenverteilungen sowie mögliche Lücken zwischen Anforderungen an den und Fähigkeiten des Nachfolgers entstehen (De Massis et al. 2008).

Auch die Beziehungen innerhalb der Familie haben Einfluss auf die Nachfolge (Collins 2011; Davis und Harveston 1998). Dabei sind sowohl die Beziehung zwischen Übergeber/in und Übernehmer/in bzw. die Eltern-Kind-Beziehung (De Massis et al. 2008; Muskat und Zehrer 2017) wie auch Beziehungen zur restlichen Familie (De Massis et al. 2008; Ghee et al. 2015; Zhou et al. 2016) miteingeschlossen. Innerhalb der Familienbeziehungen spielen Faktoren wie Zusammenhalt, Kontrolle (Zhou et al. 2016), Mitsprache, Hilfsbereitschaft, Zusammenarbeit (Steier und Miller 2010), Abhängigkeiten (Miller et al. 2003) oder Familienkultur und damit in Verbindung stehend Harmonie und Kommunikation eine wichtige Rolle (Sund et al. 2015).

Weiters ist die Planung der Übergabe an sich in den Vordergrund der Untersuchungen gerückt (Sharma et al. 2003; Chrisman et al. 2005). Getz und Petersen (2004) 
beschäftigen sich vorwiegend mit Übergabeprozessen in Familienunternehmen und den daraus resultierenden und häufig auftretenden intrafamiliären Konflikten, denn der kommunikative Austausch zwischen den Subsystemen (d.h. Beziehung zwischen Eltern, Kindern, Vater-Tochter, Mutter-Sohn, Mutter-Kindern, etc.) basiert auf angelernten und regelmäßigen Verhaltensweisen der Familienmitglieder (Oberlechner 2008). Zehrer und Haslwanter (2010) zeigen Gründe für einen misslungenen Nachfolgeprozess auf, wohingegen Spelsberg und Weber (2012) Erfolgsfaktoren für einen Nachfolgeprozess innerhalb der Familie darlegen. Die Bedeutung der Ko-Evolution in Unternehmerfamilien im Wandel, die interdependente Entwicklung der Akteure, der Beziehungssysteme und der Strukturen wird von Leiß und Zehrer (2018a) beleuchtet. Zellweger et al. (2012) konzentrieren sich auf das Unternehmertum und wie dadurch ein höherer Wert in Familienunternehmen generiert werden kann.

Dyer (2003), Lansberg und Astrachan (1994) oder Miller et al. (2003) untermauern vorrangig die emotionalen Faktoren der Übergeber-Nachfolger Beziehung und die damit verbundenen komplexen sozialen Beziehungen in Familienunternehmen. Der Wechsel der Generationen stellt viele Familienunternehmen auch vor die große Problematik, dass kein passender Nachfolger gefunden werden kann, oder dass zu viele Nachfolger für eine Position vorhanden sind (Huber und Sterr-Kölln 2006; Baumgartner 2009; Mueller-Harju 2013). Da ein Generationenwechsel nicht von heute auf morgen vonstatten geht, ist dieser als dynamischer Prozess zu sehen, der als schleichende Entwicklung beginnt und einer detaillierten Planung bedarf. Idealerweise wird der Nachfolgeprozess über einen längeren Zeitraum hinweg gewollt gesteuert (Dyck et al. 2002; Sharma 2004; Riedel 2011). Nachfolgeplanung wird damit zum essenziellen Erfolgsfaktor für das langfristige Überleben und das Wachstum von Familienunternehmen (Martin et al. 2002).

Ein weiterer Punkt der Beeinflussung entsteht innerhalb des Unternehmenskontextes durch sogenannte Kontextfaktoren. Dazu zählen bspw. Veränderungen in der Markt- und Geschäftsentwicklung (De Massis et al. 2008), potenzielle oder bestehende Unternehmenskrisen (Miller et al. 2003), Veränderungen im Wettbewerbsumfeld (Miller et al. 2003; Collins 2011), im Bereich der Schlüsselkundinnen/en (Davis und Harveston 1998; De Massis et al. 2008; Collins 2011) oder sonstigen Stakeholdern (Sund et al. 2015), in der Unternehmensperformance (Miller et al. 2003) sowie in der Organisation, Wertevorstellung oder Kultur im Unternehmen (Davis und Harveston 1998; Miller et al. 2003; Lang-von Wins und Kaschube 2007; Collins 2011). Ebenso Einfluss haben äußere Rahmenbedingungen und somit indirekte Faktoren wie gesellschaftliche Veränderungen, neue Entwicklungen, Werte oder Fähigkeiten (Ahrens et al. 2015; Breuninger 1998) sowie neue Technologien zur Unterstüt- zung des Unternehmens, auch innerhalb des Nachfolgeprozesses bspw. beim Wissenstransfer (Bracci und Vagnoni 2011). So spricht Klages (Klages 1984; Klages und Gensicke 2006a, 2006b) von der Problematik des gesellschaftlichen Wertewandels und beschreibt die Entwicklung von Pflicht- und Akzeptanzwerten (Konventionalismus wie Disziplin, Gehorsamkeit, Pflichterfüllung, Treue, Fleiß, Bescheidenheit) hin zu Selbstentfaltungswerten (Idealismus wie Emanzipation, Demokratie, Autonomie; Hedonismus: Genuss, Abenteuer, Abwechslung), wodurch Menschen immer weniger bereit sind, autoritativ geltend gemachte $\mathrm{Au}$ ßenanforderungen hinzunehmen. Auch dies hat eine Auswirkung auf die Nachfolgeproblematik.

Vor dem Hintergrund der bisherigen Überlegungen muss im Nachfolge-Coaching die Bewusstseinsbildung und die Entwicklung reflexiver Kompetenz der Akteure im Mittelpunkt stehen, denn nur im intergenerativen Dialog wird es gelingen, die strategischen Vorteile und Stärken der Unternehmerfamilie bestmöglich zu nutzen (Habbershon und Williams 1999; Habbershon et al. 2003; Kraus et al. 2011; Mühlebach 2012; Weismeier-Sammer et al. 2013).

\section{Das Fallbeispiel ***Hotel Alpenglühen}

Familienunternehmen sind prägend in manchen Branchen, so auch im Tourismus. Die Tourismusbranche ist mit etwa 5,2\% aller Arbeitskräfte in Europa und ca. 9,7 Mio. Arbeitsplätzen der drittgrößte sozioökonomische Faktor in der Europäischen Union (EU Business 2009). Das Gelingen des Übergabeprozesses beeinflusst daher nicht nur die Entwicklung des Familienunternehmens sondern auch die Entwicklung der Tourismusdestinationen, in die diese Betriebe eingebettet sind (Russell und Murphy 2005). Familienbetriebe in touristischen Destinationen sind aufgrund ihrer Historie authentische Gastgeber, welche für die Identität und Geschichte der Destination stehen und diese dem Gast vermitteln können (Arnoff und Ward 2011; Kaslow 2012). Sie zeigen vielfach eine tiefe Verbundenheit mit dem Ort; auch die Solidarität den Einheimischen gegenüber ist spürbar. In diesem Sinne verstehen sich Familienbetriebe als Bindeglied zwischen den Einheimischen und Gästen und sehen sich mitverantwortlich für den Erhalt der Destination als Lebensraum (Beritelli et al. 2007). Ihr Fortbestehen ist somit nicht unwesentlich für die Zukunft des Tourismus (Zehrer 2017).

Gegenständliches Familienunternehmen wird 1965 von Adolf und Johanna Hofer als Landgasthof mit Landwirtschaft inmitten der Alpen gegründet. Die erste Generation übergibt den Betrieb 1998 an die einzige Tochter Patrizia, die den Landgasthof mit sechs Mitarbeitern zusammen mit ihrem Mann Paul Hörmann übernimmt, den sie in einer Hotelfachschule kennenlernt. Gemeinsam entwickeln bei- 
de den Betrieb zu einem auf Familien spezialisierten, gut etablierten 4 Sterne Hotelbetrieb. 2017 erwirtschaftet das Unternehmen einen Umsatz von $€ 14$ Mio. und beschäftigt mittlerweile 134 Mitarbeiter, davon fünf Abteilungsleiter. Die Mitarbeiterfluktuation liegt unter $20 \%$, was für die Branche beachtlich ist (AMS 2016). Die Tourismusdestination, in der das Familienunternehmen angesiedelt ist, stellt mittlerweile eine erfolgreiche Ganzjahresdestination im Herzen der Tiroler Alpen dar. Der Hauptquellmarkt des Hotelbetriebes ist Deutschland, gefolgt von Österreich, der Schweiz und Großbritannien. Zu- und Umbauten prägen den Betrieb, die letzte Investition mit einer Summe von $€$ 13 Mio. erfolgt zwischen 2016 und 2017 in einen neuen Wellnessbereich sowie in die Renovierung der Zimmer.

Patrizia und Paul haben zwei Kinder, Kurt und Peter, die beide, gemeinsam mit ihren Ehepartnern Susanne und Olivia Interesse zeigen, das Familienunternehmen in den nächsten fünf Jahren zu übernehmen. Kurt, 33, besucht eine renommierte Hotelfachschule in der Schweiz, wo er auch seine Frau Susanne, 32, kennenlernt. Auch Susanne bringt viel Erfahrung in der Touristik mit. Sie haben bereits zwei kleine Kinder im Alter von ein bzw. drei Jahren. Beide arbeiten seit mittlerweile fünf Jahren im Familienunternehmen mit. Kurt hat die operative Führung inne, Susanne arbeitet vorwiegend an der Rezeption und kümmert sich um Personalangelegenheiten. Als Kurt und Susanne im Ort ihre Hochzeit feiern, verkündet der Vater vor versammelter Gemeinde, dass er den Betrieb nun an den Sohn übergeben wird. Kurt und seine Frau verlieren jedoch den Glauben an die angekündigte Übergabe, da sich diese seit fünf Jahren nicht einstellt. Kurt hat im Laufe der letzten Jahre einige Konflikte mit seinem Vater ausgetragen, da dieser immer wieder seine Arbeit kritisiert und ihm kein Vertrauen entgegenbringt. Auch was die Führung des Betriebes anlangt, haben Paul und sein Sohn Kurt unterschiedliche Vorstellungen. Während der Vater einen sehr autoritären Führungsstil praktiziert, möchte Kurt die Mitarbeiter partizipativ führen und legt viel Wert auf die Meinung der Abteilungsleiter. Kurt kritisiert zudem die fehlende Regelung der Verantwortlichkeiten im Betrieb, v. a. im Hinblick auf die Kompetenzen, die er und seine Frau aufgrund ihrer fundierten Ausbildung mitbringen und die ständigen Einmischungen seines Vaters in die Betriebsführung. Auch für Susanne sind die fehlenden Aufgabenverteilungen sowie Arbeitsplatzbeschreibungen schwierig zu handhaben; aus ihrer Sicht herrscht mangelnde Professionalität im Hinblick auf den hohen Mitarbeiterstab im Betrieb. Sie fühlt sich überlastet und nicht wertgeschätzt. Aufgrund der zahlreichen Konflikte kommunizieren die beiden Generationen fast ausschließlich via Email miteinander; die persönliche Kommunikation zwischen Vater und Sohn hat sehr stark abgenommen, was eine sehr große emotionale Last für Mutter
Patrizia darstellt, und auch die Ehe von Kurt und Susanne auf eine harte Probe stellt.

Der zweite Sohn, Peter, 27, studiert in den USA Finanzmanagement und arbeitet bei internationalen Unternehmen, wo er auch seine 30-jährige Frau Olivia, eine Südamerikanerin, kennenlernt, die in einem großen Konzern in einer führende Marketingfunktion tätig ist. Beide sind bisher nicht in den Familienbetrieb integriert, und da Peter im Ausland zur Schule gegangen ist und studiert hat, kennt auch er das Hotel lediglich vom jährlichen Skiurlaub bzw. anderen Besuchen zu familiären Anlässen. Olivia ist selbst Tochter eines Familienunternehmens, hat sich aber bewusst gegen einen Einstieg in den Familienbetrieb entschieden; daher stellt die Integration in den Familienbetrieb ihres Mannes Peter nun eine große Herausforderung dar, der sie mit Respekt begegnet, wissend, was es heißt, in einem Familienunternehmen zu arbeiten. Peter und Olivia heiraten im September 2017 und ziehen in den kleinen Alpenort, was für Olivia ein Kulturschock ist, da sie immer nur in Großstädten gelebt hat und nun in einer 1000 Seelen Gemeinde lebt. Zudem spricht sie kaum Deutsch, was ebenfalls eine Herausforderung darstellt.

Die beiden Söhne entwickeln sich beruflich komplett unterschiedlich, gehen beide auf unterschiedliche Internate und wachsen daher nicht zusammen auf. Daher sind beide auch sehr distanziert zueinander. Kurt und Peter sind zudem sehr unterschiedliche Charaktere, während Kurt sich sozial stark engagiert (er ist Mitglied bei der freiwilligen Feuerwehr sowie bei anderen Vereinen im Dorf), ist Peter ein Sportenthusiast und ein sehr ich-zentrierter Mensch. Da Peter bereits als Kleinkind an Rheuma leidet, wird er in den Augen von Kurt immer von den Eltern bevorzugt; darum hat Kurt seinen jüngeren Bruder stets beneidet. In den Augen von Kurt ist Peter der Lieblingssohn seines Vaters; während er ein angespanntes Verhältnis zu seinem Vater hat, verstehen sich Peter und Paul sehr gut. Da beide Söhne den Betrieb übernehmen möchten, fürchtet Kurt die Bevorzugung seines jüngeren Bruders.

Die Ausganglage zeigt bereits, dass es im Familienunternehmen einige Herausforderungen zu bewältigen gilt, die aufgrund des anstehenden Generationenwechsels noch verschärft werden. $\mathrm{Zu}$ den wesentlichen Themen zählen die mangelnde intergenerationale Kommunikation bzw. Gesprächskultur im Unternehmen sowie fehlende Steuerungsmechanismen (Organigramm, klare Abgrenzung von Verantwortlichkeiten) aber auch eine fehlende Family Governance (z.B. eine Familienverfassung, ein Familienrat, etc.) im Unternehmen. 


\section{Systemisches Nachfolge-Coaching in Familienunternehmen}

Business-Coaching wird als dyadischer Prozess zwischen Klient und Coach beschrieben, mit dem Ziel arbeitsbezogene Anliegen aufzuarbeiten (Feldman und Lankau 2005; Theeboom et al. 2014; Schermuly und Graßmann 2016; Schulz 2016). Coaching von Unternehmerfamilien ist grundsätzlich dem klassischen Business-Coaching ähnlich, jedoch gilt es, die Besonderheiten des Familienunternehmens $\mathrm{zu}$ berücksichtigen. „Coaching practice in family businesses has distinct features because coaching practice is designed to include specific features of a functional family, such as family values, family ethics, traditions, interpersonal relations, emotions, communication patterns, and leadership style. No other coaching type has such a diverse range of issues to deal with in a coaching intervention“" (Shams 2011, S. 9). Die Kunst im Coaching von Unternehmerfamilien liegt daher darin, allen Familienmitgliedern die entsprechende Aufmerksamkeit zu geben. Coaching wird nur dann wirksam, wenn sich der Coach der komplexen Ausgangslage in Unternehmerfamilien bewusst ist und die Dynamiken eines Familienunternehmens versteht (Lippmann 2013).

Die Schwierigkeit im Coaching liegt hierbei zudem darin, nicht nur die Familie, sondern auch die Familie an der Schnittstellte zum Unternehmen entsprechend zu fokussieren, d.h. Coaching muss sich auf beide Systeme beziehen - das System der Familie und das System des Unternehmens. Damit wird das Coaching von Unternehmerfamilien zu einer komplexen Aufgabe, die das Etablieren neuer Kommunikationsformate im Zusammenspiel der Subsysteme erfordert (Schreyögg 2011; Schwertl 2016). „As family businesses tend to give priority to socio-emotional issues (Gómez-Mejía et al. 2007), we can expect that their coaching processes will differ from those of non-family firms" (Utrilla und Torraleja 2013). Ausgehend vom Business-Coaching Ansatz und aufgrund der Besonderheiten eines Familienunternehmens muss das systemische Coaching von Unternehmerfamilien sowohl die familiären als auch die unternehmerischen Belange berücksichtigen.

„Die Praxis systemischer Organisationsberatung bedeutet Interdisziplinarität. Sie verlangt die Integration verschiedener Wissenschaftsrichtungen im Aufbau einer angemessenen Theorie des eigenen Tuns" (Wimmer 1992, S. 82). In der Prozessbegleitung eines Familienunternehmens sollten für alle im folgenden skizzierten Gesprächsformate, in sämtlichen Gesprächen mit einzelnen Familienmitgliedern sowie mit der Gesamtfamilie zwei Coaches parallel vertreten sein. Durch eine Coaching-Dyade unterliegen „die in den Gesprächen vermittelten Inhalte einer besonderen Form von Kontrolle“ (Kühl 2009, S. 68). Wichtig ist dabei, die besondere Sicherheit einer Dyade bei Wahrung der Ver- traulichkeit hervorzuheben (Simmel 1917). Die persönliche face-to-face-Kommunikation mittels der Dyade wird um ein Vielfaches flexibler und aussagekräftiger, da Gestik und Mimik es ermöglichen, durch beide Coaches bestimmte Sachverhalte und Zusammenhänge genauer zu erfassen und zu interpretieren. Im Sinne einer kollegialen Validierung können die Ergebnisse gemeinsam reflektiert werden (Steinke 1999). Was die interdisziplinäre Zusammensetzung der Dyade anlangt, ist ein Coach mit familientherapeutischer Kompetenz und ein betriebswirtschaftlicher Berater sinnvoll, die die Unternehmerfamilie gemeinsam durch die mit Abstand kritischste Phase in ihrem Lebenszyklus begleiten.

„Nachfolge-Coaching ist eine auf die spezifischen Bedürfnisse eines oder mehrerer Familienmitglieder bezogene Beratung, Betreuung und Unterstützung bei Entscheidungsund Veränderungssituationen im Zusammenhang mit der Unternehmensnachfolge" (Mueller-Harju 2013, S. 129). Bis auf wenige Autoren wird das Coaching von Unternehmerfamilien in der Literatur allerdings wenig diskutiert und stellt eine Forschungslücke dar. Dieser Beitrag unternimmt den Versuch, auf Basis des beschriebenen Fallbeispiels eine Vorgehensweise für das Nachfolge-Coaching sowie angewandte Coaching-Methoden zur Diskussion zu stellen. Nachfolgendes Vorgehensmodell hat zum Ziel, die Kontakt-, Orientierungs- und Analysephase (Rauen 2005) eines systemischen Nachfolge-Coachings in Unternehmerfamilien zu skizzieren. Dabei werden die einzelnen Prozessschritte zeitlich logisch strukturiert und um die entsprechenden Coaching-Methoden sowie Analyse-Methoden ergänzt. Das Vorgehensmodell soll als organisatorisches Hilfsmittel für ein Nachfolge-Coaching von Unternehmerfamilien dienen (Abb. 1). Dabei kommen drei unterschiedliche Coaching-Gesprächsformate zum Einsatz - Einzelgespräche, Paargespräche sowie Familienworkshops - die nachfolgend kurz theoretisch und in Abschn. 6 in ihrer exemplarischpraktischen Umsetzung erläutert werden.

Da jedes Subsystem auf Unternehmensebene aus Individuen besteht, ist primär das Individuum das grundlegende Analyseobjekt (Kast und Rosenzweig 1992; Riordan und Riordan 1993; Pieper und Klein 2007). Zunächst bedarf es daher individueller Auftragsklärungsgespräche mit allen beteiligten Stakeholdern der Unternehmerfamilie (Übergeber- und Übernehmergeneration sowie die jeweiligen Ehepartner), um die Zielklärung im Gesamt-Coachingprozess zu gewährleisten (Wastian und Poetschki 2016). Basierend auf der Zielklärungstheorie nach Locke und Latham (2002) und der klientenzentrierten Gesprächsführung nach Rogers (2005) soll die Ziel- und Auftragsklärung zunächst mit allen Akteuren face-to-face im Rahmen von Einzelgesprächen geführt werden, um die Ausgangssituation mit den Symptomen und der Entwicklungsgeschichte zu verstehen und damit verbundene Optimierungspotenziale in Bezug auf individuelle Lebensplanung, innerbetriebliche/-familia- 
Abb. 1 Vorgehensmodell zum systemischen Nachfolge-Coaching in Unternehmerfamilien

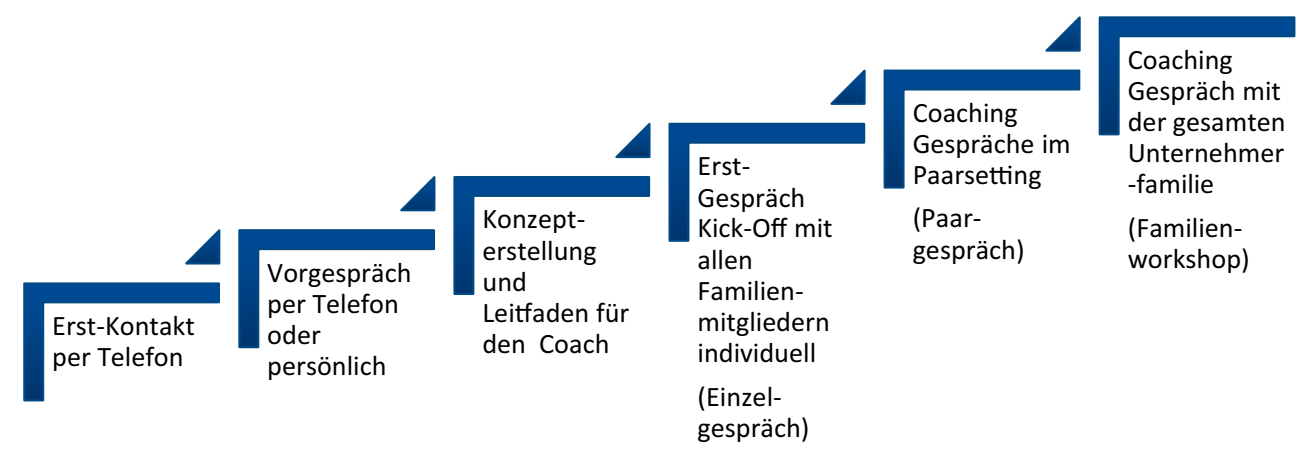

le Kommunikation, Prozesse, Rollen und Verantwortlichkeiten, Führung, organisationale Entwicklung, Innovation und Nachfolgeplanung etc. zu klären. Diese Gespräche werden am Flipchart dokumentiert und im Anschluss in Anlehnung an das Prinzip der systemischen Schleife (Königswieser und Hillebrand 2017) interkollegial zu Arbeitshypothesen verdichtet.

In einem weiteren Schritt werden Paargespräche geführt, da die Rolle der jeweiligen Partner im Familienbetrieb wichtig ist. Essentiell hierbei ist, dass ein Raum geöffnet wird, um über belastende Themen in der Unternehmerfamilie und in der Paarbeziehung zu sprechen, die eine Auswirkung auf das Gesamtsystem haben. „Was bei Paaren ohnehin schon oft zum Problem wird, kann sich bei gemeinsamer Arbeit für den Familienbetrieb verschärfen: Wer leistet mehr? Wessen Arbeit wird höher bewertet? Wie viel Zeit bekomme ich für mich? - Die Belastung der Frauen mit Haushalt und Kindererziehung ist auch heute noch in aller Regel größer als die der Männer, entsprechend bleibt weniger Zeit für das Unternehmen“ (Borst 2008, S. 218).

Der letzte Schritt sind gemeinsame Familienworkshops mit allen beteiligten Stakeholdern; LeMar $(2001,2011)$ beschreibt den Familienworkshop als Format, bei welchem alle Familienmitglieder einer Unternehmerfamilie an einem oder mehreren aufeinanderfolgenden Tage zusammenkommen, als in den meisten Fällen geeignete Maßnahme. „Die menschlich-familiären Aspekte der Unternehmensnachfolge stehen im Zentrum, die Beziehungsaspekte und emotionalen Faktoren in der Unternehmerfamilie bekommen ihren Platz“ (LeMar 2011, S. 224). Innerhalb eines ersten Familienworkshops können folgende Coaching-Tools eingesetzt werden:

- Zielarbeit und Ökologie-Check: Konstruktive W-Fragen helfen beim Herausarbeiten von Zielen, um so die individuelle Grundhaltung besser zu verstehen sowie jene der anderen Familienmitglieder nachvollziehen zu können. Folgende Fragen können nützlich sein: Was genau will ich erreichen? Woran werde ich erkennen, dass das Ziel erreicht ist? Was treibt mich an? Bis wann soll das Ziel erreicht sein? Was ist der Vorteil des Alten und des Neuen? Was sind die Konsequenzen für mich und für andere?

- Visionsarbeit (Wunderfrage oder Trance-Induktion): Diese Methode dient zur Entwicklung eines Wunschbildes für die Zukunft im und mit dem Familienbetrieb. Folgende Fragen können nützlich sein: Wie und wo sehe ich mich im Unternehmen? Was würde ich mir wünschen in Bezug auf meine Position im Unternehmen?

- Nachfolge-Landkarte (Selbsteinschätzung durch Kartenabfrage): Diese Methode dient der Verdeutlichung von Schwerpunktthemen, die die Beteiligten als vorrangig in Bezug auf den Nachfolgeprozess erachten; als mögliche Grundstruktur bietet sich das Vier-Quadranten-Modell nach Ken Wilber (2001) an, mit dem sich sowohl individuelle als auch kollektive Nachfolge-Herausforderungen im Innen und Außen einordnen lassen. Quadrant 1 bezieht sich auf individuelle Werte, Gefühle und Haltungen, Quadrant 2 auf individuelle Kompetenzen und Wissen, Quadrant 3 auf die kollektive Team-, Führungsund Unternehmenskultur in Form von Vision, Mission und Werten, und Quadrant 4 auf die kollektiven Systemstrukturen wie Strategie, Aufbauorganisation, Prozesse, Systeme und Produkte des Unternehmens.

\section{Systemisches Nachfolge-Coaching anhand des Fallbeispiels}

Nachfolgend werden exemplarisch anhand des gegenständlichen Fallbeispiels die drei Coaching-Gesprächsformate Einzelgespräche, Paargespräche sowie Familienworkshops - im Detail erläutert.

Die Einzelgespräche in gegenständlichem Fallbeispiel dauern zwischen zwei und drei Stunden. Bei Patrizia, 57, zeigt sich, dass sie bereit ist für den Ruhestand und zur Übergabe des Betriebs an die beiden Söhne, sich jedoch Sorgen macht, dass ihr Mann, Paul, 61, nicht loslassen kann und kein Vertrauen in die zukünftige Arbeit der Kinder hat. Kurt, der bereits im Betrieb mitarbeitet, stößt immer wieder an seine Grenzen in der Zusammenarbeit mit seinem Vater, der viele Informationen zurückhält, Entscheidungen nach 
wie vor alleine fällt und ihm das Gefühl gibt, er wäre mit der Arbeit überfordert. Seine Frau Susanne hat ebenfalls Herausforderungen mit dem Schwiegervater zu meistern; diese liegen v.a. in der Tatsache, dass sie mit Arbeit überhäuft wird und für Tätigkeiten, die normalerweise zwei Mitarbeiter erledigen, alleine verantwortlich ist, nach dem Motto „das haben wir früher schon so gemacht“; Susanne beklagt vor allem die Tatsache, dass sich die Betriebsgröße von früher auf heute verhundertfacht hat. Peter, der mit seiner Frau Olivia in den Alpenort ziehen wird und ebenfalls plant, in den Betrieb einzusteigen, weiß, dass Kurt ihn fürchtet und schätzt sich gegenüber seinem Bruder als durchaus überlegen ein. In den Einzelgesprächen lassen sich präferierte Kommunikations- und Verhaltensmuster der jeweiligen Familienmitglieder erkennen. Intergenerative Konflikte zwischen Paul und Kurt werden nicht direkt besprochen, die betriebliche Kommunikation erfolgt primär über Email.

Die Paargespräche in gegenständlichem Fallbeispiel dauern jeweils ca. zwei bis drei Stunden. Für die Seniorgeneration, Patrizia und Paul, stellt sich die Situation wie folgt dar: Nach der Eheschließung hat Paul, der ursprünglich aus Deutschland kommt, das Hotel seiner Schwiegereltern zu dem gemacht, was es heute ist. Er bezeichnet sich selbst als „mittellosen Stifter“, der für das in einer Privatstiftung befindliche Vermögen seiner Frau verantwortlich zeichnet und die noch bestehenden Schulden bis 2035 zurückbezahlen möchte. Innerhalb der Familie übernimmt er die Rolle des „Richters“, der die finanzielle Zukunft für sich und seine Frau absichern muss. Er trennt Familie und Unternehmen strikt und sieht eine Betriebsübergabe nur durch einen radikalen Schnitt. Er bezeichnet sich selbst als „Statthalter“, ist sich aber unklar, wann seine Rolle enden soll. Seinen beiden Söhnen - im Einzelgespräch nennt er sie „Fremdkörper im Betrieb" - traut er die eigenverantwortliche Hotelführung nicht zu. Auch eine familienexterne Übergabe ist für ihn vorstellbar. Da sein Lebensinhalt in den letzten 30 Jahren darin bestand, das Hotel auf- und auszubauen, blieb kaum Zeit für Freundschaften oder Hobbies. Im Innersten hat er Angst, nach dem Rückzug wie sein Schwiegervater in ein Loch zu fallen. Patrizia sitzt zwischen den Stühlen und leidet unter den andauernden Streitereien zwischen ihrem Mann und der jungen Generation. Da sie die Rolle der „Schlichterin“ übernommen hat, ist sie mittlerweile auch gesundheitlich angeschlagen und wünscht sich nichts sehnlicher, als dass Paul und sie sich aus dem Hotel zurückziehen, um mehr Zeit für ihre Enkelkinder und Reisen zu haben. Eine gemeinsame Vision, wie ihr Leben nach dem Ausstieg aus dem Unternehmen aussehen soll, haben beide nicht. Daher ist beim Seniorpaar nach wie vor das weitere Berufs- und Privatleben nach der Betriebsübergabe ein wesentliches Thema. Hier hat sich gezeigt, dass viel Unsicherheit und Angst besteht. Zudem stellte sich die
Frage des künftigen Wohnortes, da die Privatwohnung des Seniorpaars im Hotelbetrieb angesiedelt ist.

Beim Juniorpaar, welches bereits im Betrieb arbeitet, kommen Themen zur Sprache wie die fehlende Kommunikation und das fehlende Vertrauen, fehlende klare Vorgaben im betrieblichen Ablauf und in der Organisationsstruktur sowie die daraus resultierenden Diskussionen, die beim Ehepaar immer wieder zu schwierigen Situationen in ihrer Beziehung führen. Auch die Rolle der Enkelkinder wird angesprochen. Das Juniorpaar, welches erst ins Unternehmen einsteigt, hat viel Respekt vor der gemeinsamen Arbeit, wissend, dass es bereits zahlreiche Herausforderungen zwischen den vier derzeit im Betrieb integrierten Familienmitgliedern gibt (strategische Ausrichtung, Zeitpunkt der Übergabe, mangelnde Kommunikation, etc.).

In gegenständlichem Fallbeispiel werden auf Grundlage der Einzel- und Paargespräche zentrale Arbeitshypothesen aufgestellt, die bei der Vorbereitung und Durchführung des Familienworkshops eine wichtige Rolle spielen. So zeichnet sich klar ab, dass der intergenerative Konflikt zwischen Paul und dem im Betrieb mitarbeitenden Übernehmer-Paar Kurt und Susanne bereits weiter fortgeschritten, aber noch in keiner Weise bearbeitet wurde und deshalb im Familienworkshop sichtbar werden würde. Auch ist davon auszugehen, dass die Familienmitglieder wenig Übung im Verstehen der jeweils anderen Perspektiven haben, und die individuellen Erwartungen und Gefühle bezüglich der Nachfolge unbedingt offengelegt werden sollten. Der hier exemplarisch dargestellte erste Familienworkshop dauert fünf Stunden mit einer zwanzig-minütigen Pause. Nach einer grundlegenden Klärung der Gesprächs- und Verhaltensregeln für den Workshop werden die Erwartungen für den Familienworkshop besprochen. Im Anschluss werden die vier Ebenen von organisationalen Change-Prozessen erhoben. Hier zeigt sich, dass bei allen Familienmitgliedern zunächst ganz stark individuelle Werte, Gefühle und Haltungen thematisiert werden und die kollektive Ebene im Sinne einer gemeinsamen Unternehmenskultur und/oder -struktur kaum angesprochen wird. Die Coaches ergänzen deshalb Expertentipps oder geben Impulse zu Fragen zeitgemäßer unternehmerischer Führung, bezogen auf Personalmanagement, Organisationsdesign und Übergabe-Strategie. Die Vorbereitung, Durchführung und Nachbereitung des Familienworkshops mit allen Stakeholdern der Unternehmerfamilie hat das Ziel, ein von allen getragenes unternehmerisches $\mathrm{Zu}$ kunftsbild sowie einen maßgeschneiderten Maßnahmenplan für die Betriebsübergabe zu entwickeln. Zunächst stehen die Herstellung von Commitment zur Weiterentwicklung der Aufbau-Organisation, der Kommunikationskultur sowie die Vereinbarung konkreter Aufgaben (Klärung der Aufgaben- und Kompetenzprofile aller Familienmitglieder, Einführung regelmäßiger Meetings zur innerbetrieblichen Informationsweitergabe und Abstimmung) im Vordergrund. 
Mit Hilfe von systemischen und zirkulären Fragen können die Coaches erschließen, wie das Familien- und Unternehmenssystem sich für jedes einzelne Familienmitglied darstellt (von Schlippe und Schweitzer 1996). Die damit erreichte Metaebene ermöglicht es den Coaches, andere Beobachtungsstandpunkte einzunehmen und andere Sichtweisen zu erkennen. Dadurch konnten letztlich die Wahrnehmungs- und Beschreibungsfähigkeit der Familienmitglieder erweitert, neue Blickwinkel auf sich selbst und das Unternehmen ermöglicht, sowie Denkprozesse nach alternativen Lösungsansätzen initiiert werden (Ellebracht et al. 2003).

\section{Fazit und Ausblick}

Das auf Basis eines Fallbeispiels entwickelte Vorgehensmodell für Nachfolge-Coaching arbeitet in der Kontakt-, Orientierungs- und Analysephase mit Einzel- und Paargesprächen sowie mit Familienworkshops. Bei allen Formaten besteht das übergeordnete Ziel darin, systemimmanente Kommunikationsparadoxien zu thematisieren, konfliktträchtige Nachfolgethemen zu bearbeiten und mit allen betroffenen Familienmitgliedern mögliche Maßnahmen auf der individuellen und kollektiven Ebene abzuleiten. Da jede Unternehmerfamilie ihre ureigenen Themen zu bewältigen hat, empfiehlt sich, wie bei allen Coachingprozessen, auch hier ein prozesshafter Beratungsansatz, der Interventionen im Sinne der systemischen Schleife entwickelt und adaptiert (Königswieser et al. 1995; Königswieser und Exner 2001). Dadurch gehen die Coaches in ,den Kreislauf zwischen Diagnose, Hypothesenbildung und Veränderungsimpulsen in Gestalt von Reflexion und Metakommunikation“ (Jarmai und Königswieser 1992, S. 261).

Das erarbeitete Vorgehensmodell beschreibt einen Prozess für ein Nachfolge-Coaching und gibt damit einen Rahmen vor, in dem die Klärung zentraler Übergabe-Themen geordnet und strukturiert ablaufen kann. Das Vorgehensmodell kann den Erfolg der Übergabe jedoch nicht garantieren. Die Auswahl und Konzeption geeigneter Gesprächsformate bleibt Aufgabe der Coaches. Darüber hinaus muss auch sichergestellt sein, dass die am Nachfolgeprozess beteiligten Personen über den gesamten Zeitraum zur Verfügung stehen, was bei einem komplexen Vorhaben wie der Betriebsnachfolge nicht immer einfach ist. Die Qualität des Übergabeprozesses bleibt primär von den beteiligten Personen, ihrer Teamfähigkeit und ihrem Commitment abhängig. In gegenständlichem Fallbeispiel hat die Zusammenarbeit zwischen Coaches und der Unternehmerfamilie sehr gut funktioniert. Somit sprechen trotz Kritik zahlreiche Argumente für die Praxistauglichkeit des entwickelten Vorgehensmodells. Nichtsdestotrotz kann das Vorgehensmodell flexibel an den jeweiligen Anwendungsfall angepasst werden.
Aus Sicht der Coaches war zu beobachten, dass sich erarbeitete Themen entlang des Vorgehensmodells eher mittelund langfristig ausgewirkt haben und hier ein Vertrauen in den Prozess sehr wichtig war. Dies wurde auch von der Unternehmerfamilie bestätigt. Insgesamt wurde von der Unternehmerfamilie rückgemeldet, dass der gesamte Prozess als wirksam gesehen wurde. Im Laufe der Prozessbegleitung zeigten die Familienmitglieder zunächst großes Interesse an einer systematischen Übergabe-Planung und -gestaltung und beteiligten sich aktiv an der Analyse der Ist-Situation und der Ableitung von Verbesserungs- und Innovationspotenzialen. Jedoch war auch augenscheinlich, dass gegen Ende des im Beitrag beschriebenen Familienworkshops eine deutliche Eskalation des bis dahin latent unterdrückten intergenerativen Konflikts zwischen Übergeber und Übernehmer zu beobachten war. Dabei wurde deutlich, dass vor allem mit Blick auf die innerfamiliale Kommunikation und auf den Rollenwechsel von den jetzigen Geschäftsführern auf die nachfolgende Generation konkrete, zeitlich definierte Maßnahmen zu setzen sind. Diese können als organisationale und prozessuale Innovationen verstanden werden. Vor dem Hintergrund des doch sehr verschärften familialen Konflikts wäre eine deutlich engmaschigere Nachfolgeberatung notwendig gewesen, um weiter in die Umsetzung zu gehen.

Daher muss, ausgehend vom Business-Coaching Ansatz und aufgrund der Besonderheiten eines Familienunternehmens, das systemische Coaching von Unternehmerfamilien sowohl die familiären als auch die unternehmerischen $\mathrm{Be}$ lange berücksichtigen. Eine Coaching-Dyade mit einem Coach mit familientherapeutischer Kompetenz und einem betriebswirtschaftlichen Berater ermöglicht systemische Interventionen auf allen Ebenen und gewährleistet einen hohen Qualitätsstandard im Sinne von Orientierungs-, Handlungs- und Verfahrenssicherheit (Staubach 2008). Gemeinsame Verhaltensanalysen ermöglichen es der Dyade, interpersonelle Dynamiken und soziale Interaktionen komplementär durchzuführen und dadurch Verhaltensmuster sowie zirkulären Ursache-Wirkungs-Zusammenhänge mehrperspektivisch zu erkennen (Wimmer 2001; Ianiro und Kauffeld 2012).

Wie eingangs erwähnt, verdichten sich aus gegenständlichem Fallbeispiel sowie den Erkenntnissen aus der Literatur folgende zentralen Grundannahmen, die neben dem zur Diskussion gestellten Vorgehensmodell im Nachfolge-Coaching einen wesentlichen Anhaltspunkt und analytischen Bezugsrahmen für ein systemisches Nachfolge-Coaching bieten sollen.

1. Nachfolge-Coaching wird nur dann wirksam, wenn bei den Coaches ein Bewusstsein für die komplexe Ausgangslage in Unternehmerfamilien besteht und die Dynamiken 
sowie Ursache-Wirkungs-Zusammenhänge eines Familienunternehmens verstanden werden.

2. Dabei gilt es insbesondere, neue Kommunikationsformate im Zusammenspiel der Subsysteme Familie und Unternehmen zu entwickeln. Für ein systemisches Nachfolge-Coaching in Unternehmerfamilien ist ein Mix an Gesprächsformaten zielführend, bestehend aus Einzel-, Paargesprächen sowie Familienworkshops, um allen Familienmitgliedern die entsprechende Aufmerksamkeit zu geben und der Komplexität einer Unternehmerfamilie Rechnung zu tragen.

3. Als Coaching-Methoden für die systemische Begleitung von Unternehmerfamilien bieten sich an: Zielarbeit und Ökologie-Check, Visionsarbeit (Wunderfrage oder Trance-Induktion), Nachfolge-Landkarte (Selbsteinschätzung durch Kartenabfrage).

4. Ein systemisches Nachfolge-Coaching erfordert eine kontinuierliche Prozessbegleitung durch eine CoachingDyade mit einem Coach mit familientherapeutischer Kompetenz und einem betriebswirtschaftlichen Berater, um den komplexen systemimmanenten Zusammenhängen, Verhaltensmuster und -strukturen eines Familienunternehmens bestmöglich gerecht zu werden.

Die Kunst, als Coach in ein System wirkungsvoll zu intervenieren, liegt darin, den richtigen Zeitpunkt zu finden, um Interventionen zu setzen und diese so zu gestalten, dass sie die Grenze des dem Familien- und Unternehmenssystem Zumutbaren berühren. Dadurch können die komplexen $\mathrm{Zu}-$ sammenhänge zwischen individueller Belastung, Familiendynamik und Unternehmensproblemen rechtzeitig erkannt und angegangen werden (Borst 2008). Letztlich bleibt die Betriebsübergabe die zentrale Herausforderung in Unternehmerfamilien; es gilt diese aktiv anzugehen, die nächste Generation frühzeitig einzubinden und Unterstützung im Gesamtprozess anzunehmen.

Abschließend soll auch noch auf die Limitationen des Artikels im Hinblick auf das Fallbeispiel eingegangen werden. Zunächst ist festzuhalten, dass sämtliche Aussagen zunächst fallgebunden sind, damit in ihrer Aussagekraft begrenzt und nicht universal anwendbar sind (Hering und Schmidt 2014). Schließlich zielt der Beitrag aber auf das Hinterfragen theoretischer Aussagen und auf die Gewinnung neuer Kenntnisse ab (Borchardt und Göthlich 2009); so betont Lamnek (2010), dass aus einem Fallbeispiel ein ganzheitliches Bild abgeleitet und nicht nur bestimmte Variablen betrachtet werden sollen.

Open Access Dieser Artikel wird unter der Creative Commons Namensnennung 4.0 International Lizenz (http://creativecommons.org/ licenses/by/4.0/deed.de) veröffentlicht, welche die Nutzung, Vervielfältigung, Bearbeitung, Verbreitung und Wiedergabe in jeglichem Medium und Format erlaubt, sofern Sie den/die ursprünglichen Autor(en) und die Quelle ordnungsgemäß nennen, einen Link zur Creative Commons Lizenz beifügen und angeben, ob Änderungen vorgenommen wurden.

\section{Literatur}

Ahrens, J.P., Landmann, A., \& Woywode, M. (2015). Gender preferences in the CEO successions of family firms: Family characteristics and human capital of the successor. Journal of Family Business Strategy, 6, 86-103.

AMS (2016). Arbeitsmarktlage 2016. http://www.ams.at/_docs/001_ JB-2016.pdf. Zugegriffen: 26. März 2018.

Arnoff, C.E., \& Ward, J.L. (2011). Family business values. New York: Macmillan.

Baumgartner, B. (2009). Familienunternehmen und Zukunftsgestaltung: Schlüsselfaktoren zur erfolgreichen Unternehmensgestaltung. Wiesbaden: Gabler.

Beritelli, P., Bieger, T., \& Laesser, C. (2007). Destination governance: using corporate governance theories as a foundation for effective destination management. Journal of Travel Research, 46(1), 96-107.

Borchardt, A., \& Göthlich, S. (2009). Erkenntnisgewinnung durch Fallstudien. In S. Albers, D. Klapper, U. Konradt, A. Walter \& J. Wolf (Hrsg.), Methodik der empirischen Forschung (S. 33-48). Wiesbaden: Gabler.

Borst, U. (2008). Psychische Störungen und Familienunternehmen. Die Angst des Juniors vor der Nachfolge. In A. von Schlippe, A. Nischak \& M. El Hachimi (Hrsg.), Familienunternehmen verstehen. Gründer, Gesellschafter und Generationen (S. 209-221). Göttingen: Vandenhoeck \& Ruprecht.

Bracci, E., \& Vagnoni, E. (2011). Understanding small family business succession in a knowledge management perspective. The IUP Journal of Knowledge Management, 9(1), 7-36.

Breuninger, H. (1998). Der Generationswechsel in Familienunternehmen aus psychologischer Sicht. In B.-H. Hennerkes \& R. Kirchdörfer (Hrsg.), Unternehmenshandbuch Familiengesellschaften - Sicherung von Unternehmen, Vermögen und Familie (S. 752-767). Köln: Heymann.

Brückner, C. (2011). Der Nachfolger kommt! Eine Analyse des Generationenwechsels in Familienunternehmen. München, Mering: Hampp.

Burböck, B., \& Krenn, W. (2014). Asymmetric influence on satisfaction in the takeover-process of SMEs in Austria. Advances in Business-Related Scientific Research Journal, 5(2), 99-109.

Cabrera-Suárez, K., de Saá-Pérez, P., \& García-Almeida, D. (2001). The succession process from a resource- and knowledge-based view of the family firm. Family Business Review, 14(1), 37-46.

Cater, J. J., \& Justis, R. T. (2009). The development of successors from followers to leaders in small family firms: an exploratory study. Family Business Review, 22(2), 109-124.

Chrisman, J. J., Chua, J. H., \& Sharma, P. (2005). Trends and directions in the development of a strategic management theory of the family firm. Entrepreneurship: Theory \& Practice, 29(5), 555-575.

Collins, L. (2011). To succeed or not to succeed: A multiple perspectives literature review of research in family business succession. http://eprints.uwe.ac.uk/14705/2/Collins.FOB_Succession_A_ Literature_Review.march2011.pdf IFERA 2011, 28th June-1st July.

Davis, P.S., \& Harveston, P.D. (1998). The influence of family on the family business succession process: a multi-generational perspective. Entrepreneurship Theory and Practice, 22, 31-54.

Davis, P. S., \& Harveston, P. D. (1999). In the founder's shadow: Conflict in the family firm. Family Business Review, 12, 311-323.

Dyck, B., Mauws, M., Starke, F. A., \& Mischke, G. A. (2002). Passing the baton: the importance of sequence, timing, technique, and communication in executive succession. Journal of Business Venturing, 17, 143-181.

Dyer, W. G. (2003). The family: the missing variable in organizational research. Entrepreneurship Theory and Practice, 27(4), 401-417. 
Ellebracht, H., Lenz, G., Osterhold, G., \& Schäfer, H. (2003). Systemische Organisations- und Unternehmensberatung: Praxishandbuch für Berater und Führungskräfte. Wiesbaden: Gabler.

EU (2015). Stellungnahme über Familienunternehmen in Europa. http://www.europarl.europa.eu/sides/getDoc.do?pubRef=-//EP// TEXT+REPORT+A8-2015-0223+0+DOC+XML+V0//DE． Zugegriffen: 20. März 2018.

EUBusiness (2009). Familienunternehmen wichtig für österreichische und europäische Wirtschaft. http://www.eubusiness.at/ familienunternehmen-wichtig-fur-osterreichische-und-europa ische-wirtschaft. Zugegriffen: 10. März 2018.

Feldman, D. C., \& Lankau, M. J. (2005). Executive coaching: a review and agenda for future research. Journal of Management, 31(6), 829-848.

Getz, D., \& Petersen, T. (2004). Identifying industry-specific barriers to inheritance in small family businesses. Family Business Review, 17(3), 259-276.

Ghee, W. Y., Ibrahim, M. D., \& Abdul-Halim, H. (2015). Family business succession planning: unleashing the key factors of business performance. Asian Academy of Management Journal, 20(2), $109-126$.

Goel, S., \& Jones, R. J. (2016). Entrepreneurial exploration and exploitation in family business. A systematic review and future directions. Family Business Review, 29(1), 94-120.

Gómez-Mejía, L. R., Takacs, K. H., Nunez-Nickel, M., and Jacobson, K. J. L. (2007). Socioemotional wealth and business risks in family-controlled firms: Evidence from Spanish olive oil mills. Administrative Science Quarterly, 52, 106-137.

Habbershon, T.G., \& Williams, M. (1999). A resource-based framework for assessing the strategic advantages of family firms. Family Business Review, 12(1), 1-25.

Habbershon, T.G., Williams, M., \& MacMillan, I. C. (2003). A unified systems perspective of family firm performance. Journal of Business Venturing, 18(4), 451-465.

Halter, F. A. (2009). Familienunternehmen im Nachfolgeprozess - Die Emotionen des Unternehmers. Köln: Josef Eul.

Halter, F. A., \& Schröder, R. (2011). Unternehmensnachfolge in der Theorie und Praxis. Das St. Galler Nachfolgemodell. Bern: Haupt.

Handler, W.C. (1994). Succession in family business: a review of the research. Family Business Review, 7(2), 133-158.

Harveston, P., Davis, P., \& Lyden, J. (1997). Succession planning in family business: The impact of owner gender. Family Business Review, 10(4), 373-396.

Harvey, M., \& Evans, R. (1995). Life after succession in the family business: is it really the end of problems? Family Business Review, $8,3-16$

Harvey, M., \& Evans, R. E. (1994). Family business and multiple levels of conflict. Family Business Review, 7, 331-348.

Hennerkes, B.-H., Berlin, M., \& Berlin, T. (2007). Die Familie und ihr Unternehmen in Österreich. München: FinanzBuch.

Hering, L., \& Schmidt, R. (2014). Einzelfallanalyse. In J. Blasius (Hrsg.), Handbuch Methoden der empirischen Sozialforschung (S. 529-542). Wiesbaden: Springer VS.

Huber, H.G., \& Sterr-Kölln, H. (2006). Nachfolge in Familienunternehmen - den Generationenwechsel erfolgreich gestalten. Stuttgart: Schaeffer-Poeschl.

Humphreys, M.M.C. (2013). Daughter succession: a predominance of human issues. Journal of Family Business Management, 3(1), 24-44.

Ianiro, P., \& Kauffeld, S. (2012). Wann stimmt die „Chemie“ im Coaching? Untersuchungen zur gemeinsamen „Augenhöhe“ von Coach und Klient. Coaching Magazin, 1, 44-48.

Jackson, J. (1972). Role. Sociological Studies, Bd. 4. Cambridge: Cambridge University Press.

Jarmai, H., \& Königswieser, R. (1992). Problemdiagnose. In R. Königswieser \& C. Lutz (Hrsg.), Das systemisch-evolutionäre Ma- nagement: der neue Horizont für Unternehmer (S. 258-264). Wien: Orac.

Kaslow, F. W. (2012). A handbook of family business and family business consultation-A global perspective. New York: Routledge.

Kast, F.E., \& Rosenzweig, J.E. (1992). System concepts: Pervasiveness and potential. Management International Review, 32, 40-49.

Kets de Vries, M. (1993). The dynamics of family controlled firms: the good and the bad news. Organizational Dynamics, 21, 59-71.

Klages, H. (1984). Wertorientierungen im Wandel. Rückblick, Gegenwartsanalyse und Prognosen. Frankfurt am Main: Campus.

Klages, H., \& Gensicke, T. (2006a). Wertewandel und Big-Five-Dimensionen. In S. Schumann (Hrsg.), Persönlichkeit. Eine vergessene Größe der empirischen Sozialforschung (S. 279-200). Wiesbaden: VS.

Klages, H., \& Gensicke, T. (2006b). Wertesynthese - funktional oder dysfunktional. Kölner Zeitschrift für Soziologie und Sozialpsychologie, 58(2), 332-351.

Klein, S. (2000). Family business in Germany-significance and structure. Family Business Review, 8(3), 157-182.

Klein, S. (2004). Familienunternehmen. Theoretische und empirische Grundlagen. Wiesbaden: Gabler.

Klein, B. S., Jaskiewicz, P., May, P., \& von Schlippe, A. (2010). Familienunternehmen theoretische und empirische Grundlage. Lohmar: Josef Eul.

Königswieser, R., \& Exner, A. (2001). Systemische Intervention. Stuttgart: Klett-Cotta.

Königswieser, R. \& Hillebrand, M. (2017). Einführung in die systemische Organisationsberatung. Heidelberg: Carl-Auer.

Königswieser, R., Exner, A., \& Pelikan, J. (1995). Systemische Intervention in der Beratung. Organisationsentwicklung, 2, 52-65.

Kraus, S., Märk, S., \& Peters, M. (2011). The influences of family on the everyday business of a family firm entrepreneur. International Journal of Entrepreneurship and Small Business, 12(1), 82-100.

Kühl, S. (2009). Coaching und Supervision: Zur personenorientierten Beratung in Organisationen. Wiesbaden: VS.

Lamnek, S. (2010). Qualitative Sozialforschung. Weinheim: Beltz.

Lang-von Wins, T., \& Kaschube, J. (2007). Die Psychologie des Unternehmertums. In M. Frey \& L. von Rosenstiel (Hrsg.), Wirtschaftspsychologie. Enzyklopädie der Psychologie, Serie Wirtschafts-, Organisations- und Arbeitspsychologie. (S. 149-201). Göttingen: Hogrefe.

Lansberg, I. (1988). The succession conspiracy. Family Business Review, 1(2), 119-143.

Lansberg, I., \& Astrachan, J.H. (1994). Influence of family relationships on succession planning and training: the importance of mediating factors. Family Business Review, 7, 39-59.

Lee, D. S., Lim, G. H., \& Lim, W. S. (2003). Family business succession: Appropriation risk and choice of successor. Academy of Management Review, 28(4), 657-666.

Leiß, G., \& Zehrer, A. (2018a). Intergenerational communication in family firm succession. Journal of Family Business Management. https://doi.org/10.1108/JFBM-09-2017-0025.

Leiß, G., \& Zehrer, A. (2018b). Freiheit in Verbundenheit - Ko-Evolution in Unternehmerfamilien. In M. Lueger, H. Frank \& Ch Korunka (Hrsg.), Die Unternehmerfamilie im Kontext ihres Familienunternehmens (S. 231-250). Wien: Facultas.

LeMar, B. (2001). Generations- und Führungswechsel im Familienunternehmen. Mit Gefühl und Kalkül den Wandle gestalten. Heidelberg: Springer.

LeMar, B. (2011). Familienworkshops als Weg der Entwicklung einer Familienstrategie. In A. Von Schlippe, A. Nischak \& M. El Hachimi (Hrsg.), Familienunternehmen verstehen - Gründer, Gesellschafter und Generationen (S. 223-234). Göttingen: Vandenhoeck \& Ruprecht.

Leppa, T., Plakoyiannaki, E., \& Dimitratos, P. (2016). The case study in family business. An analysis of current research practices and recommendations. Family Business Review, 29(2), 159-173. 
Lippmann, E. (2013). Coaching: angewandte Psychologie für die Beratungspraxis. Heidelberg: Springer.

Locke, E. A., \& Latham, G.P. (2002). Building a practically useful theory of goal setting and task motivation. American Psychologist, 57(9), 705-717.

Luhmann, N. (1995). Social systems. Stanford: Stanford University Press.

Martin, C., Martin, L., \& Mabbett, A. (2002). SME ownership succession-business support and policy implications. Birmingham: University of Central England.

De Massis, A., Chua, J. H., \& Chrisman, J. J. (2008). Factors preventing intra-family succession. Family Business Review, 21(2), 183-199.

Miller, D., Steier, L., \& Le-Breton-Miller, I. (2003). Lost in time: intergenerational succession, change, and failure in family business. Journal of Business Venturing, 18(4), 513-531.

Mueller-Harju, D. (2013). Generationenwechsel im Familienunternehmen: Mit Emotionen und Konflikten konstruktiv umgehen. Heidelberg: Springer.

Mühlebach, C. (2012). Family business governance. In M. Hilb (Hrsg.), Corporate Governance im Praxistest (S. 111-124). Bern: Haupt.

Muskat, B., \& Zehrer, A. (2017). A power perspective on knowledge transfer in internal succession of small family businesses. Journal of Small Business \& Entrepreneurship, 29(5), 333-350.

Mussolino, D., \& Calabró, A. (2014). Paternalistic leadership in family firms: Types and implications for intergenerational succession. Journal of Family Business Strategy, 5, 197-210.

Nordqvist, M., Wennberg, K., Bau, M., \& Hellerstedt, K. (2013). An entrepreneurial process perspective on succession in family firms. Small Business Economics, 40(4), 1087-1122.

Oberlechner, M. (2008). Jede Familie ist ein wichtiges System - Subjektive Sichtweisen der Familienrealität. Die Südtiroler Frau, 8, 14.

Pieper, T., \& Klein, S. (2007). The bulleye: A Systems approach to modeling family firms. Family Business Review, 20(4), 301-319.

Pounder, P. (2015). Family business insights. An overview of the literature. Journal of Family Business Management, 5(1), 116-127.

Prügl, R., \& Hauck, J. (2012). Deutschlands nächste Unternehmergeneration II. Eine empirische Untersuchung der Werthaltungen, Einstellungen und Pläne. https://www.familienunternehmen.de/ media/public/pdf/publikationen-studien/studien/Studie_Stiftung_ Familienunternehmen_Deutschlands-naechste-Unternehmergene ration-2015.pdf Stiftung Familienunternehmen.

Rauen, C. (2005). Handbuch Coaching. Göttingen: Hogrefe.

Riedel, C. (2011). Praxishandbuch Unternehmensnachfolge. Bonn: Zerb.

Riordan, D. A., \& Riordan, M. P. (1993). Field theory: An alternative to systems theories in understanding the small family business. Journal of Small Business Management, 31(2), 66-78.

Rogers, C.R. (2005). Die klientenzentrierte Gesprächspsychotherapie. Frankfurt: Fischer.

Rüsen, T. (2009). Krisen und Krisenmanagement in Familienunternehmen - Schwachstellen erkennen, Lösungen erarbeiten, Existenzbedrohung meistern. Wiesbaden: Gabler.

Russell, R., \& Murphy, P. (2005). Entrepreneurial leadership in times of uncertainty: implications for tourism research and education. Clevedon: Channel View Publications.

Schermuly, C.C., \& Graßmann, C. (2016). Die Analyse von Nebenwirkungen von Coaching für Klienten au einer qualitativen Perspektive. Coaching Theorie \& Praxis, 2, 33-47.

Schlepphorst, S., \& Moog, P. (2014). Left in the dark: Family successors' requirement profiles in the family business succession process. Journal of Family Business Strategy, 5, 358-371.

von Schlippe, A. (2009). Psychologie der familieninternen Nachfolge in Familienunternehmen. In R. Kirchdörfer, R. Lorz, A. Wiedemann, R. Kögel \& T. Frohnmayer (Hrsg.), Familienunternehmen in Recht, Wirtschaft, Politik und Gesellschaft (S. 39-55). München: Beck. von Schlippe, A., \& Schweitzer, J. (1996). Lehrbuch der systemischen Therapie und Beratung. Göttingen: Vandenhoeck \& Ruprecht.

von Schlippe, A., Nischak, A., \& El Hachimi, M. (2008). Familienunternehmen verstehen. In A. von Schlippe, A. Nischak \& M. El Hachimi (Hrsg.), Familienunternehmen verstehen. Gründer, Gesellschafter und Generationen (S. 19-29). Göttingen: Vandenhoeck \& Ruprecht.

von Schlippe, A., Groth, T., \& Rüsen, T. A. (2017). Die beiden Seiten der Unternehmerfamilie: Familienstrategie über Generationen. Göttingen: Vandenhoeck \& Ruprecht.

Scholes, L., Westhead, P., \& Burrows, A. (2008). Family firm succession-the management buy-out and buy-in routes. Journal of Small Business and Enterprise Development, 15(1), 8-30.

Schreyögg, A. (2011). Konfliktcoaching: Anleitung für den Coach. Frankfurt: Campus.

Schulz, F. (2016). Ein organisationstheoretischer Ansatz zur Erforschung diskursiver Prozesse im Management Coaching. Coaching Theorie \& Praxis, 2, 1-7.

Schwertl, W. (2016). Kommunikative Kompetenz im Business-Coaching: Reflexionen über eine oft missverstandene Dienstleistung. Heidelberg: Springer.

Shams, M. (2011). Key issues in family business coaching. In M. Shams \& D. A. Lane (Hrsg.), Coaching in the family owned business - a path to growth (S. 1-12). London: Kamac Books.

Sharma, P. (2004). An overview of the field of family business studies: current status and directions for the future. Family Business Review, 17(1), 1-36.

Sharma, P., Chrisman, J. J., \& Chua, J.H. (2003). Succession planning as planned behavior: some empirical results. Family Business Review, 16(1), 1-15.

Simmel, G. (1917). Grundfragen der Soziologie. Berlin: de Gruyter.

Simon, F. B. (2005). Familien und Unternehmen. Überlegungen zu Unterschieden, Gemeinsamkeiten und den Folgen. In F. B. Simon (Hrsg.), Die Familie des Familienunternehmens. Ein System zwischen Gefühl und Geschäft (S. 18-34). Heidelberg: Carl-Auer.

Simon, F. B. (2007). Einführung in Systemtheorie und Konstruktivismus. Heidelberg: Carl-Auer.

Simon, F. B. (2009). Organisationen und Familien als soziale Systeme unterschiedlichen Typs. In A. von Schlippe (Hrsg.), Beiträge zur Theorie des Familienunternehmens (S. 17-45). Lohmar: Josef Eul.

Simon, F. B., Wimmer, R., \& Groth, T. (2005). Mehr-Generationen-Familienunternehmen. Erfolgsgeheimnisse von Oetker, Merck, Haniel u. a. Heidelberg: Carl-Auer.

Sipos, W. (2012). Wenn Lehrer wieder zu Lernenden werden - Die Rollenvielfalt aus dem Blickwinkel Studierender eines Lehramtstudiums in der Berufspädagogik. Soziologie heute, 4, 19-22.

Spelsberg, H., \& Weber, H. (2012). Familieninterne und familienexterne Unternehmensnachfolgen in Familienunternehmen im empirischen Vergleich. BFuP - Betriebswirtschaftliche Forschung und Praxis, 64(1), 73-97.

Stadler, C. \& Kern, S. (2010). Psychodrama - eine Einführung. Wiesbaden: VS

Staubach, M. (2008). Co-Produktion - Ein Entwurf zur Konzeptionierung von Coaching. ZSTB, 26(1), 6-13.

Steier, L. P., \& Miller, D. (2010). Pre- and post-succession governance philosophies in entrepreneurial family firms. Journal of Family Business Strategy, 1(1), 145-154.

Steinke, I. (1999). Kriterien qualitativer Forschung. Ansätze zur Bewertung qualitativ-empirischer Sozialforschung. Weinheim: Juventa.

Sund, L. G., Melin, L., \& Haag, K. (2015). Intergenerational ownership succession-Shifting the focus from outcome measurements to preparatory requirements. Journal of Family Business Strategy, 6, 166-177.

Tagiuri, R., \& Davis, J. A. (1996). Bivalent attributes of the family firm. Family Business Review, 9(2), 199-208. 
Theeboom, T., Beersma, B., \& Van Vianen, A.E. M. (2014). Does coaching work? A meta-analysis on the effects of coaching on individual level outcomes in an organizational context. The Journal of Positive Psychology, 9(1), 1-18.

Utrilla, P. N.-C., \& Torraleja, F. A. G. (2013). The importance of mentoring and coaching for family businesses. Journal of Management \& Organization, 19, 386-404.

Wastian, M., \& Poetschki, J. (2016). Zielklärung und Zielerreichung im Coaching. Ergebnisse einer qualitativen Untersuchung von Coaching-Prozessen. Coaching Theorie \& Praxis, 2(1), 21-31. https://doi.org/10.1365/s40896-016-0011-3.

Weismeier-Sammer, D., Frank, H., \& von Schlippe, A. (2013). Untangling "familiness". A literature review and directions for future research. The International Journal of Entrepreneurship and Innovation, 14(3), 165-177.

Wilber, K. (2001). Integral psychology. Consciousness, spirit, psychology, therapy. Boston: Shambhala.

Wimmer, R. (1992). Organisationsberatung. Neue Wege und Konzepte. Wiesbaden: Gabler.

Wimmer, R. (2001). Organisationsberatung - eine ,unmögliche“ Dienstleistung. In T.M. Bardmann \& T. Groth (Hrsg.), Organisation, Management und Beratung. Zirkuläre Positionen, (Bd. 3, S. 197-220). Wiesbaden: Westdeutscher Verlag.
Wimmer, R., Groth, T., \& Simon, F.B. (2009). Erfolgsmuster von Mehrgenerationen-Familienunternehmen. In A. von Schlippe (Hrsg.), Beiträge zur Theorie des Familienunternehmens (S. 95-171). Lohmar: Josef Eul.

Wiswede, G. (1977). Rollentheorie. Stuttgart: Kohlhammer.

Zehrer, A. (2017). Touristische Familienunternehmen als tragende Säule des Alpentourismus - Zentrale Herausforderungen für Unternehmertum und Tourismuspolitik. In P. Bußjäger \& Ch Gsodam (Hrsg.), Tourismus als treibende Kraft für regionale Kooperation im Alpenraum (S. 47-61). Wien: New Academic Press.

Zehrer, A., \& Haslwanter, J. (2010). Management of change in tourism-the problem of family internal succession in family-run tourism SMEs. Electronic Journal of Family Business Management, 2(4), 147-162.

Zellweger, T.M., Kellermanns, F.W., Chrisman, J.J., \& Chua, J.H. (2012). Family control and family firm valuations by family CEos: the importance of intentions for transgenerational control. Organization Science, 23(3), 851-868.

Zhou, Y., Hu, Q., Yao, J., \& Qin, X. (2016). The determinants of family business owners' intrafamily succession intention: An interplay between business owners and institutional environment. Chinese Management Studies, 10(4), 710-725. 